\title{
HPV: 2-Dosen-Impfschema wirkt
}

— Eine HPV-Impfung kann hochgradige Zervixläsionen und -karzinome verhindern. Verfügbar ist zum Beispiel der Impfstoff Cervarix $^{\circledast}$ gegen die HPV-Typen 16 und 18. Für den Impfstoff wurde außerdem eine Kreuzprotektion gegen die weiteren Risikotypen HPV 33, 31 und 45 nachgewiesen [Lancet Oncol 2012;13:100-10]. Wie Prof. Dr. Tino Schwarz vom Krankenhaus der Stiftung Juliusspital in Würzburg erläuterte, erklärt sich durch diesen zusätzlichen protektiven Effekt auch die hohe Gesamtwirksamkeit des Impfstoffs: $64,9 \%$ (CIN2+) beziehungsweise $93,2 \%$ (CIN3+) in der Gruppe der Impf-naiven Kohorte [Lancet Oncol 2012;13:89-99]. Diese Gesamtwirksamkeit sei deutlich höher als der Anteil der durch HPV 16 und 18 zusammen verursachten Läsionen: $51 \%$ der CIN2/3-Läsionen beziehungsweise $71 \%$ der Zervixkarzinome [Lancet Oncol 2010;11:1048-56].

Bereits 2014 wurde Cervarix ${ }^{\circledR}$ im 2-DosenImpfschema zugelassen. Basis der Zulassung war unter anderem die Studie HPV048, welche die Nichtunterlegenheit des 2-Dosen-Impfschemas bei Mädchen bis 14 Jahre gegenüber des 3-Dosen-Impfsche- mas bei Mädchen und Frauen zwischen 15 und 25 Jahren zeigte: Die Antikörperkonzentration war in beiden Gruppen über einen Zeitraum von vier Jahren nahezu identisch, erklärte Schwarz [Hum Vaccin Immunother 2014;10:1155-65]. Seit Anfang 2015 kann der HPV-Impfstoff flexibel verabreicht werden: die zweite Dosis im Abstand von fünf bis 13 Monaten nach der ersten Dosis. Durch das 2-Dosen-Impfschema kann schon früh eine höhere Durchimpfungsrate bei Mädchen erreicht werden, fasste Schwarz zusammen.

Entsprechend der Empfehlung der Ständigen Impfkommission (STIKO) wurde die Schutzimpfungsrichtlinie des Gemeinsamen Bundesausschusses (G-BA) aktualisiert und das Alter für die HPV-Impfung herabgesetzt: Das 2-Dosen-Impfschema gilt damit als Standardschema für Mädchen zwischen 9 und 14 Jahren. Für diese Altersgruppe werden die Impfkosten von den gesetzlichen Krankenkassen übernommen.

Ann Köbler

Symposium „HPV-Impfung: was gibt es Neues?"; Düsseldorf, 6. März 2015; Veranstalter: GlaxoSmithKline

\section{Neue Therapieoption bei schwerer Psoriasis}

— Bei etwa einem Drittel aller PsoriasisPatienten - rund 1,6 Millionen in Deutschland - beginnt die Erkrankung bereits in der Kindheit, berichtete Dr. Uwe Schwichtenberg, niedergelassener Dermatologe in Bremen. Eine frühzeitige Diagnose und Therapie ist besonders wichtig, weil die körperlichen und emotionalen Belastungen der Betroffenen mit zunehmender Krankheitsdauer kumulieren. Auch das kardiovaskuläre Risiko und die Mortalität von Psoriasis-Patienten sind erhöht.

Die Diagnosestellung einer Psoriasis ist allerdings für Nicht-Dermatologen nicht immer einfach. Neben sehr charakteristischen Befunden wie Hautläsionen an den Streckseiten der Ellenbogen oder im Gesicht können zum Beispiel auch Hände oder Füße betroffen sein, berichtete Schwichtenberg. Er empfahl eine enge Zusammenarbeit von Pädiatern, Hausärzten und Dermatologen, die jeweils zu etwa einem Drittel Kinder und
Jugendliche mit Psoriasis versorgen. Die Behandlung sollte abgestuft nach dem Schweregrad der Psoriasis und nach dem individuellen Behandlungsziel erfolgen.

Die Therapieoptionen bei Kindern mit schwerer Plaque-Psoriasis waren bisher begrenzt. Für Kinder ab 4 Jahren mit schwerer chronischer Plaque-Psoriasis ist seit kurzem Adalimumab (Humira ${ }^{\circledR}$ ) zugelassen. „Der TNF-Blocker zählt zu den am besten wirksamen Medikamente bei Psoriasis", sagte Prof. Dr. Matthias Augustin, vom Universitätsklinikum Hamburg-Eppendorf. Zudem verringern sich unter einer Therapie mit TNF-Blockern auch häufige psychiatrische Begleitsymptome der Patienten wie Depressionen und Ängste. Roland Fath

Pressegespräch „Pädiatrische Psoriasis Versorgungssituation und aktuelle Therapieoptionen vor dem Hintergrund des Risikos lebenslanger, kumulierender Belastungen"; Berlin, 29. April 2015; Veranstalter: AbbVie

\section{Jahre Hustenkompetenz}

Seit 65 Jahren ist Prospan ${ }^{\circledast}$ sowohl bei akuten als auch bei chronisch-entzündlichen Atemwegsinfekten mit der Begleiterscheinung Husten ein Mittel der Wahl. Die Wirksamkeit und Verträglichkeit des Efeu-Spezial-Extraktes EA $575^{\circledR}$ konnte bis heute in zahlreichen Studien und Untersuchungen nachgewiesen werden. Das zeigt etwa auch die Veröffentlichung von Lang et al. [Planta Med 2015;81:96874]. Hier fassen die Autoren mehr als 65.000 Patientendaten zusammen und zeigen dabei die therapeutische Relevanz von $E A 575^{\circ}$ auf: Vom Einsatz in allen Altersklassen über Wirksamkeit und Verträglichkeit auch im Vergleich zu chemisch-definierten Präparaten. Ergänzt wird diese Datenlage nun um die Ergebnisse einer aktuellen Studie (über 1.000 Schulkinder, 6-12 Jahre mit akuter Bronchitis), die die Wirksamkeit und Verträglichkeit von Prospan ${ }^{\circledR}$ erneut belegt.

Nach Informationen von Engelhard Arzneimittel

\section{Macrogol-Laxans ab 6 Monaten}

Mit Laxbene ${ }^{\circledast}$ junior steht seit Kurzem eine neue Therapieoption für Säuglinge und Kinder im Alter zwischen 6 Monaten und 8 Jahren zur Behandlung einer funktionellen Obstipation zur Verfügung. Es wird im Magen-Darm-Trakt weder resorbiert noch metabolisiert. Der bewährte Wirkstoff Macrogol hat keinen Einfluss auf den Stoffwechsel, sodass kein Gewöhnungseffekt eintritt. Laxbene ${ }^{\circledast}$ junior ist rezeptfrei erhältlich und bis zum Patientenalter von 8 Jahren erstattungsfähig.

Nach Informationen von Recordati Pharma

FSME lauert auch im eigenen Garten

Zwei besondere Aspekte gilt es bei der Frühsommer-Meningoenzephalitis (FSME) zu beachten: Zum einen ist die Erkrankung nicht nur ein Problem des Frühsommers, denn sie hat einen zweiten Inzidenzgipfel von September bis Oktober. Zum anderen sind Zecken nicht nur in Wäldern, Feldern und Wiesen, sondern insbesondere auch in Gärten weit verbreitet. Diese neuen Erkenntnisse könnten mehr Menschen als bisher dazu motivieren, sich gegen FSME impfen zu lassen. FSME-IMMUN 0,25 ml Junior ${ }^{\otimes}$ für Kinder im Alter von 1 bis 15 Jahren ist dafür ein gut verträglicher Impfstoff.

Nach Informationen von Pfizer 